\title{
HIGHLIGHTS
}

URINARY TRACT OBSTRUCTION

\section{Metal stents for malignant ureteral obstruction}

Metal mesh stents are a viable option for long-term decompression of the upper urinary tract in select patients with malignant ureteral obstruction. So say Evangelos Liatsikos and colleagues from the University of Patras, Greece, who report their 10-year experience with these devices in The Journal of Urology.

During 1996-2005 the investigators percutaneously placed expandable metal

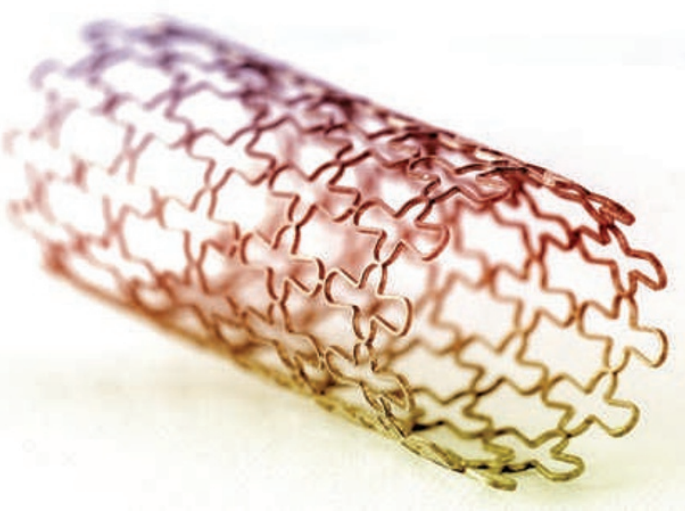

stents in a total of 34 men and 56 women with unilateral $(n=61)$ or bilateral $(n=29)$ ureteral obstruction and renal failure or evidence of deteriorating renal function. All stents were placed through a nephrostomy tract, under fluoroscopic guidance, and in the setting of antibiotic prophylaxis. Two or more overlapping stents were used when needed to bridge long obstructed ureteral segments. Patients underwent follow-up examination at 1, 3, 6 and 12 months after stent implantation, and annually thereafter.

Percutaneous stent placement was successfully achieved in all cases; elevated serum creatinine levels and hydronephrosis resolved within 1-2 weeks after the procedure.

\& Perioperative complications included mild flank pain $(n=41)$, irritative bladder symptoms $(n=5)$ and recurrent urinary tract infection $(n=3)$.

Patients were followed up for a median of 15 months after stent insertion.
Within 3 months, decreased ureteral patency owing to hyperplastic reaction and encrustation was seen in 39 and 6 stented ureters, respectively. In addition, stent migration to the bladder occurred in 13 ureters. Thus, the overall primary patency rate was $52.2 \%$. Secondary interventions were unsuccessful in 45 cases, necessitating double-pigtail or external-internal stent insertion, and representing a secondary patency rate of $62.1 \%$.

Overall, then, metal stents provided long-term ureteral patency in a good proportion of cases of malignant ureteral obstruction. The use of drug-eluting stents in this setting might further improve outcomes, and merits evaluation.

Nick Groves-Kirkby

Original article Liatsikos, E. N. et al. Ureteral metal stents: 10-year experience with malignant ureteral obstruction treatment. J. Urol. 182, 2613-2618 (2009) 